\title{
Focus on: le spondiloartriti. La diagnosi precoce può modificare il decorso della malattia?
}

\section{Focus on the spondyloarthritides. Can earlier diagnosis change the course of the disease?}

\section{Domenico Galasso $^{\mathrm{a}, *}$, Giovanni Forte ${ }^{\mathrm{b}}$, Norma Marigliano ${ }^{\mathrm{c}}$}

\author{
a Direttore Emerito di Medicina Interna, Azienda Ospedaliero "Pugliese-Ciaccio", Catanzaro \\ ${ }^{\mathrm{b}}$ Specialista in Malattie dell'Apparato Digerente \\ "Facoltà di Farmacologia, Università degli Studi "Magna Graecia", Catanzaro
}

Ricevuto il 27 marzo 2010; accettato il 7 maggio 2010

disponibile online il 5 agosto 2010

\section{KEYWORDS \\ Enthesitis; \\ Dactylitis; \\ Human Leukocyte \\ Antigen B-27 (HLA-B27); \\ Rheumatoid Factor; \\ Magnetic Resonance \\ Imaging; \\ Pelvis and sacro-iliac \\ joint.}

\begin{abstract}
Summary The spondyloarthritides (or spondyloarthropathies) (SPAs) are chronic, inflammatory, rheumatic diseases of unknown origin, which share certain clinical, epidemiological, and genetic characteristics. They include ankylosing spondylitis, reactive arthritis (also known as the Reiter Syndrome), psoriatic arthritis, enteropathic spondyloarthropathy (ulcerative colitis, Crohn's disease), undifferentiated spondyloarthritis, juvenile spondyloarthritis, and formes frustes such as acute anterior uveitis, spondyloarthritic carditis, and balanitis circinata. In the past, the SPAs were considered variants of rheumatoid arthritis, but it is now clear that they differ from the latter disease in terms of the pattern of articular and extra-articular involvement, their lack of association with seropositivity for rheumatoid factor, and their strong association with sacro-iliac joint bacino= pelvis sacro-iliac joint sacro-iliac joint sacro-iliac joint sacro-iliac joint sacro-iliac joint the class I human leukocyte antigen B27. sacro-iliac joint bacino= pelvis sacro-iliac joint sacro-iliac joint sacro-iliac joint sacro-iliac joint sacro-iliac joint Their general characteristics are axial involvement; enthesitis; peripheral arthritis involving the lower limbs, which is usually asymmetric; dactylitis; extra-articular manifestations involving the skin, eyes, bowel, and genitals. The musculoskeletal manifestations of the SPAs are due to inflammation at the level of the entheses. It is important to distinguish between the numerous clinical SPA variants based on analysis of symptoms, laboratory tests, and instrumental studies. Thanks to a greater understanding of the pathogenesis of the SPAs and the widespread availability of highly sensitive imaging modalities for their diagnosis, it is now possible to identify these diseases early and modify their course with effective therapy. This approach offers benefits to patients in terms of reduced morbidity and mortality and improved quality of life.

(c) 2010 Elsevier Srl. All rights reserved.
\end{abstract}

* Corrispondenza: Azienda Ospedaliera "Pugliese-Ciaccio", via V. Cortese 10 - 88100 Catanzaro.

E-mail: domenico.galasso@libero.it (D. Galasso). 


\section{Introduzione}

Le spondiloartriti o spondiloartropatie (SpA) sono un gruppo di malattie reumatiche infiammatorie croniche, a eziologia sconosciuta, che condividono caratteristiche cliniche, epidemiologiche e genetiche. Sebbene in passato siano state considerate varianti di artrite reumatoide, esse si distinguono da quest'ultima per differenti pattern di coinvolgimento articolare ed extrarticolare, per l'assenza del fattore reumatoide sierico e per una forte associazione con l'antigene di istocompatibiltà di classe I HLA-B27 [1-4].

\section{Aspetti clinici}

I quadri clinici comprendono: la spondilite anchilosante (SA), l'artrite reattiva (sindrome di Reiter) (ARe), l'artropatia psoriasica (APs), l'artropatia enteropatica (colite ulcerosa o malattia di Crohn), le spondiloartriti indifferenziate, le spondiloartriti giovanili, le forme fruste come l'uveite anteriore acuta, la cardite spondiloartritica e la balanite circinata [5-8] (tabella 1).

Caratteristiche generali sono: l'interessamento assiale, l'entesopatia, l'artrite periferica, prevalente a carico degli arti inferiori, spesso asimmetrica, la dattilite, le manifestazioni extrarticolari (cute, occhio, intestino, genitali). Le manifestazioni muscolo-scheletriche delle SpA sono la conseguenza dell'infiammazione, tipica delle SpA, che si realizza a livello delle entesi, delle articolazioni sacroiliache, dello scheletro assile, delle articolazioni degli arti inferiori e di alcune strutture non articolari come l'intestino, la cute, l'occhio e le valvole cardiache [9-12].

Tipica è la flogosi delle entesi e, secondariamente, della membrana sinoviale e dell'osso, che comporta quadri clinici artropatici "polimorfi". Si definisce entesi l'area di inserzione di tendini, legamenti, capsule articolari e fasce sulla superficie ossea. Si riconoscono due tipi di entesi:

- entesi fibrose (tessuto connettivo fibroso denso, che lega il tendine o il legamento all'osso; metafisi e diafisi delle ossa lunghe);

- entesi fibrocartilaginee (nei siti di maggiore attività articolare) costituite da quattro zone: estremità del tendine (tessuto connettivo fibroso denso), fibrocartilagine non calcificata (fibroblasti e condrociti), fibrocartilagine calcificata, osso [13-16].

Tabella 1 Classificazione delle spondiloartriti.

Entità cliniche degli anni Novanta

- Spondilite anchilosante primitiva (AS)

- Artrite psoriasica (APs)

- Artrite enteropatica associata a malattia infiammatoria intestinale (rettocolite ulcerosa e malattia di Crohn)

- Artrite reattiva (ARe)

- Forme indifferenziate (uSpA)

- Forme giovanili

- Forme fruste: uveite anteriore acuta, cardite spondiloartritica, balanite circinata

Prevalenza delle spondiloartriti: 1-2\%

Forme più frequenti.
L'entesite esprime un processo infiammatorio non specifico, con marcata proliferazione capillare e modesti infiltrati di linfociti, plasmacellule e (meno frequentemente) polimorfonucleati. L'entesite ha sedi articolari ed extrarticolari come: le articolazioni sinoviali sacroiliache e interapofisarie; le articolazioni cartilaginee, comprendenti la giunzione discovertebrale, la sinfisi pubica, l'articolazione manubriosternale; le sindesmosi come il legamento interosseo delle articolazioni sacroiliache; infine le entesiti periferiche extrarticolari, come l'achillea, la fascite plantare, le inserzioni muscolo-tendinee pelviche [17-20].

Può essere presente dattilite, che appare come una tumefazione globale "a salsicciotto" di una o più dita, delle dita delle mani, e dei piedi, dovuta a una tenosinovite dei flessori.

Il fattore reumatoide e gli anticorpi aticitrullina (antiCCP) sono assenti. Significative sono l'aggregazione familiare e l'associazione con gli antigeni di istocompatibilità di classe I (HLA-B27) [21-24] (tabella 2).

L'artropatia psoriasica e le artriti enteropatiche, pur facendo parte del gruppo delle SpA, possono presentare varianti cliniche senza coinvolgimento dell'asse. Le entesiti sono pressoché costantemente rilevabili in tutti i membri del gruppo. Il termine "artroentesite" si adatta meglio di "spondiloartrite" alla realtà clinica.

Le forme indifferenziate (uSpA), o limitate, possono presentarsi come: oligopoliartrite sieronegativa degli arti inferiori, dattilite, entesite achillea, sacroileite in fase "early" (senza modificazioni rilevabili alla radiografia). Sono molto diffuse e, secondo alcuni autori [25-28], possono arrivare sino al $43 \%$.

L'HLA-B27 fornisce il più forte contributo genetico allo sviluppo delle SpA. La frequenza fenotipica del B27 è nel Sud Europa tra il 2 e il $6 \%$, a fronte del $25-40 \%$ delle popolazioni esquimesi. Il $90 \%$ circa di questi individui non svilupperà mai una SpA. Nei soggetti con SpA, la prevalenza varia dal $50 \%$ della spondiloartropatia psoriasica al $90 \%$ della spondilite anchilosante.

Il rischio di SpA tra i parenti di primo grado di pazienti spondilitici è all'incirca del $30 \%$.

Esistono 25 differenti sottotipi di HLA-B27 (da HLA-B*2701 a HLA-B*2725). I due sottotipi maggiormente associati alla SpA nella popolazione bianca sono HLA-B*2705 e l'HLA$B^{*} 2702$; $i$ meno associati sono HLA-B*2706 in Asia e HLAB*2709 in Sardegna.

Gli altri geni chiamati in causa, in aggiunta al B27, sono quelli che conferiscono suscettibilità alla psoriasi (PSORS1 sul

Tabella 2 Caratteristiche generali delle spondiloartriti.

Interessamento assiale

Entesopatia

Artrite periferica (arti inferiori, spesso asimmetrica)

Dattilite

Manifestazioni extrarticolari (cute, occhio, intestino, genitali)

Assenza del fattore reumatoide e degli anticorpi anticitrullina (anti-CCP)

Aggregazione familiare significativa

Associazione con gli antigeni di istocompatibilità di classe I (HLA-B27) 
cromosoma 6p) e alle malattia di Crohn (NOD2 sulla regione pericentromerica del cromosoma 16) [29-30].

\section{Caratteristiche del dolore infiammatorio e manifestazioni cliniche}

Le caratteristiche cliniche della lombalgia infiammatoria sono: esordio subdolo, dolore sordo, difficile da localizzare, spesso intermittente e unilaterale, all'inizio più intenso a riposo, associato a rigidità all'inizio del movimento, attenuato dal movimento, accentuato dai colpi di tosse e dagli starnuti, astenia. Caratteristica è la sciatica mozza, la cui peculiarità è di essere alternante, espressione dell'interessamento delle articolazioni sacroiliache.

L'artrite periferica ha le seguenti caratteristiche: è oligoarticolare, asimmetrica e predilige le grosse articolazioni degli arti inferiori [31-34].

Le uSpA possono esordire con una dattilite per tumefazione globale "a salsicciotto" di una o più dita, oppure con entesiti del tendine achilleo o della fascia plantare [35-38]. Può tuttavia esistere un interessamento extrascheletrico sotto forma di uveite anteriore acuta, insufficienza aortica associata a blocchi di branca, fibrosi polmonare (apici), infiammazione intestinale aspecifica, amiloidosi (in fase avanzata).

L'uveite ha proprie caratteristiche cliniche: è anteriore, monolaterale, ricorrente, non granulomatosa, ha una durata di 1-6 mesi e si ha una guarigione con restitutio ad integrum.

Le manifestazioni cardiache vanno dall'insufficienza aortica ai disturbi della conduzione (blocchi atrioventricolari, blocchi di branca, blocchi fascicolari), all'insufficienza mitralica, all'interessamento miocardico. Il coinvolgimento polmonare può portare all'interessamento delle gabbia toracica, alla malattia fibrobollosa dell'apice polmonare, alla malattia polmonare interstiziale.

Il dolore infiammatorio ha una sensibilità del 71-75\% e una specificità del 75-80\%; l'entesite una sensibilità del 16$37 \%$ e una specificità dell' $89-94 \%$; l'artrite periferica una sensibilità del 40-62\% e una specificità del 90-92\%; la dattilite una sensibilità del $12-24 \%$ e una specificità del 96-98\%; la familiarità ha una sensibilità del 7-36\% e una specificità del 93-99\%; l'uveite una sensibilità del 10-22\% e una specificità del 97-99\%; la risposta ai FANS una sensibilità del 61-67\% e una specificità dell'80-85\%; il B27+ una sensibilità dell'83-96\% e una specificità del 90-96\%; la RMN(STIR) una sensibilità del $90 \%$ e una specificità del 90\% [39] (tabella 3).

\section{Decorso e progressione della malattia}

Si passa dalla perdita della lordosi lombare e all'atrofia dei glutei, all'accentuazione della cifosi toracica, all'incurvamento in avanti, se è interessata la colonna cervicale, al coinvolgimento dell'anca, che porta a contratture in flessione, con conseguente flessione compensatoria del ginocchio.

Manifestazioni in fase avanzata sono: la sublussazione atloepistrofea, l'osteoporosi del rachide con possibili fratture, la sindrome della coda equina, la spondilodiscite, nel $5-18 \%$ dei casi, la coxite, l'anchilosi del rachide [40].
Tabella 3 Diagnosi di spondilite anchilosante e spondiloartrite.

\begin{tabular}{lccc}
\hline Manifestazione & $\begin{array}{c}\text { Sensibilità } \\
(\%)\end{array}$ & $\begin{array}{c}\text { Specificità } \\
(\%)\end{array}$ & LR \\
\hline Dolore infiammatorio rachideo & $71-75$ & $75-80$ & 3,1 \\
Entesite & $16-37$ & $89-94$ & 3,4 \\
Artrite periferica & $40-62$ & $90-92$ & 4,0 \\
Dattilite & $12-24$ & $96-98$ & 4,5 \\
Familiarità & $7-36$ & $93-99$ & 6,4 \\
Uveite & $10-22$ & $97-99$ & 7,3 \\
Risposta ai FANS & $61-77$ & $80-85$ & 5,1 \\
B27+ & $83-96$ & $90-96$ & 9,0 \\
RMN(STIR) & 90 & 90 & 9,0
\end{tabular}

Legenda: LR = Likelihood ratio: Sensibilità/(100 - Specificità). Fonte: modificata Rudwaleit M, et al. Ann Rheum Dis 2004;63(5): 535-43.

\section{Fattori prognostici}

La prognosi è peggiore in presenza di HLA-B27 positivo, sesso maschile, indici di flogosi elevati, giovane età, basso livello socioeconomico, malattia già evoluta alla diagnosi, rapida evoluzione.

\section{Criteri classificativi e criteri diagnostici della spondiloartrite}

Esistono alcune differenze: i criteri diagnostici si applicano al singolo paziente e hanno alta sensibilità; i criteri classificativi si applicano a gruppi di pazienti e hanno alta specificità. Entrambi i criteri utilizzano caratteristiche cliniche, di laboratorio e di imaging delle SpA. Il picco di incidenza della SA è tra i 12 e i 45 anni ed esiste un notevole ritardo diagnostico tra la comparsa dei sintomi e la diagnosi.

\section{Analisi dei vari criteri}

I criteri clinici di Calin comprendono:

- esordio prima dei 40 anni;

- andamento graduale e insidioso, prevalentemente notturno, cronico (durata di almeno 3 mesi);

- associazione con rigidità mattutina $>60^{\prime}$, miglioramento con l'esercizio;

- assenza di miglioramento con il riposo.

Il test si considera positivo se sono presenti 4 dei 5 criteri elencati [6,41-43].

I criteri di New York modificati per AS (1984) comprendono sia criteri clinici, come:

- dolore lombare e rigidità per più di 3 mesi, che migliorano con l'esercizio ma non con il riposo,

- limitata motilità della colonna lombare, nei piani sagittale e frontale,

- limitazione dell'espansione toracica, rispetto ai valori normali, correlati per età e sesso, sia il criterio radiologico:

- sacroileite di grado $\geq 2$ bilateralmente o di grado 3-4 unilateralmente.

L'AS è definita se il criterio radiologico è associato ad almeno 1 criterio clinico. 
L'impegno può essere asimmetrico in fase iniziale.

Nel grado 1 e 2 si ha perdita di definizione della rima, con aree di atrofia nel tratto inferiore, erosioni superficiali, sclerosi focale della corticale. Nel grado 3, si estendono le erosioni nella corticale con immagini di allargamento dell'interlinea, cui seguono sclerosi subcondrale ileale, proliferazione ossea, ponti ossei, con obliterazione della rima. Nel grado 4 si hanno scomparsa della rima, eburnizzazione diffusa e anchilosi completa (tabella 4, fig. 1).

I criteri di Amor hanno una sensibilità del $90 \%$ e una specificità dell' $86,6 \%$. Si attribuisce un punteggio da 1 a 2 per ciascun disturbo. La diagnosi di SpA è possibile se lo score è $\geq 6$ [42].

Anche i criteri di Berlino hanno ribadito il ruolo dei seguenti sintomi: rigidità mattutina $>30$ ', miglioramento con l'esercizio e non con il riposo, dolore gluteo alternante, risveglio per il dolore solo nella seconda metà della notte $[44,45]$.

I criteri classificativi dello European Spondyloarthropatty Study Group (ESSG) ribadiscono il criterio clinico a impegno assiale o periferico, l'impegno radiologico con una sensibilità dell'86\% e una specificità dell'87\%, mentre nei casi "early" solo del $68 \%$ e $93 \%$, rispettivamente.

Le raccomandazioni ASAS/EULAR 2005, rivolte a soggetti con dolore infiammatorio, età $<45$ anni e durata della sintomatologia $>3$ mesi, hanno posto in primo piano due possibilità:

- la diagnosi strumentale di sacroileite associata a uno dei seguenti segni clinici: dolore infiammatorio, artrite, entesite, uveite, dattilite, psoriasi, malattia di Crohn o colite ulcerosa, buona responsività ai FANS, storia familiare di SpA, HLA-B27, elevati valori di proteina C-reattiva;

- oppure HLA-B27 con altri due sintomi di SpA, dolore infiammatorio lombare e sacroileite, identificata mediante RMN, o sacroileite dimostrata radiologicamente, in accordo con i criteri di New York modificati $[46,47]$.

I criteri ASAS (2009) confermano il valore di:

- età all'esordio < 40 anni;

- esordio insidioso;

- miglioramento con l'esercizio;

- assenza di miglioramento con il riposo;

- dolore notturno.

Se presenti nella misura di 4 su 5 , hanno una sensibilità del $79,6 \%$ e una specificità del 72,4\% [44,48]. Le probabilità diventano più alte se presenti:

Tabella 4 Grading della sacroileite radiologica (1966).

Grado $0 \quad$ Normale

Grado 1 Sacroileite sospetta (modificazioni non definite)

Grado 2 Sacroileite minima (definita come perdita della definizione ai margini delle SI, piccole aree localizzate con minima erosione o sclerosi, assente o possibile, e alterazione dell'ampiezza articolare)

Grado 3 Sacroileite moderata (con sclerosi definita su entrambi i lati, margini offuscati o indistinti, modificazioni erosive, perdita dello spazio articolare)

Grado 4 Sacroileite severa (fusione completa o anchilosi delle articolazioni)

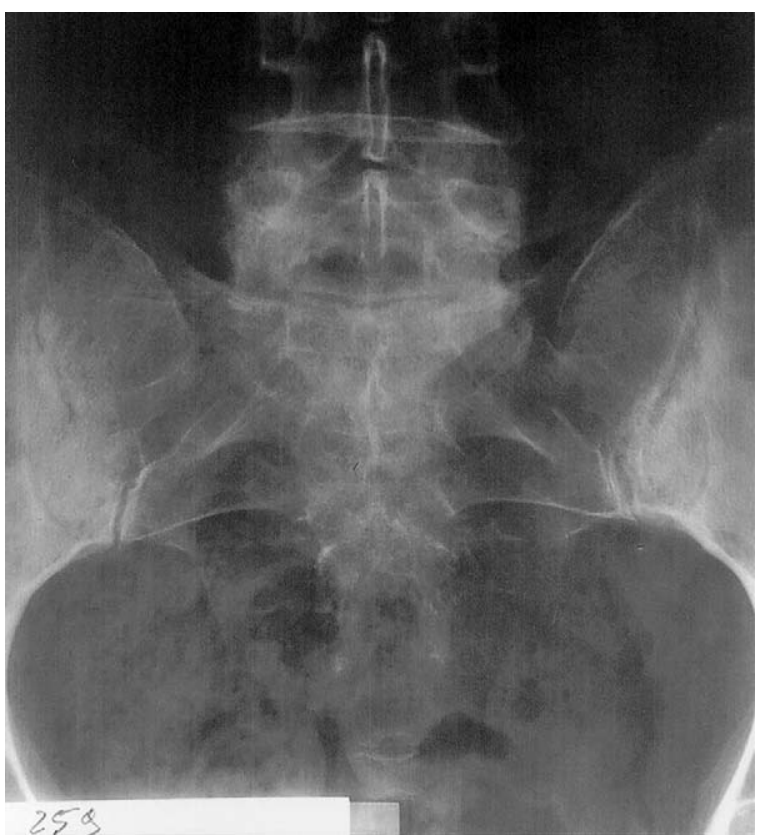

Figura 1 Sacroileite bilaterale.

Fonte: Marigliano N, Galasso D. Spondiloartrite sieronegativa indifferenziata con familiarità per psoriasi e malattia infiammatoria intestinale. Sindrome sicca. Italian Journal of Medicine 2010 (in press).

- caratteristiche cliniche;

- dati di laboratorio;

- imaging delle SpA assiali.

Per esempio: entesite calcaneare, familiarità, HLA-B27, sacroileite evidenziata con radiografia o RMN.

Le forme indifferenziate o limitate possono evolvere in forme differenziate. In una casistica di 68 pazienti con uSpA (criteri ESSG) [29], l'evoluzione a 2 anni è stata la seguente:

- forme ancora indifferenziate $75 \%$;

- remissione $13 \%$;

- evoluzione in AS 10\%;

- evoluzione in APs $2 \%$.

Le glutalgie e la positività di HLA-B27 sono talora fattori associati a progressione in forma definita. Le uSpA possono essere considerate una forma early di AS.

I criteri classificativi di Wright e Moll escludono i pazienti con uSpA. I pazienti con uSpA sono inclusi nei criteri classificativi di Amor (1990) ed ESSG (1991). Sono criteri classificativi e non diagnostici, con limiti, specie per le forme precoci (meno di 1 anno).

\section{Spondiloartriti: criticità}

Le SpA sono talvolta considerate benigne, con outcome favorevole. Potenzialmente possono causare una severa disabilità. I pazienti con SA severa presentano grave disabilità, come i pazienti con artrite reumatoide di pari età. Esistono limiti diagnostici; infatti, l'intervallo di tempo tra l'esordio del dolore rachideo infiammatorio e l'evidenza di sacroileite radiologica può variare da 5 a 10 anni. Di qui la necessità di sviluppare criteri di precocità diagnostica, anche al di fuori del riconoscimento di una sacroileite radiologica [49-52]. 
Il ritardo nella diagnosi di SA è dovuto al fatto che occorrono anni dall'inizio del dolore infiammatorio rachideo perché si manifesti la sacroileite radiologicamente definita. Ciò è da ricondursi a varie cause: dalla comparsa relativamente tardiva della sacroileite radiologica, criterio diagnostico decisivo [53-55], alla difficoltà nel differenziare fra sacroileite di grado 1 , sospetta, e sacroileite di grado 2, malattia definita, al basso grado di conoscenza di AS/SpA fra i non reumatologi.

Il gruppo ASAS (Assessment of SpondyloArthritis international Society) ha meglio definito il carattere del dolore infiammatorio rachideo e ha identificato nella RMN lo strumento di precocità infiammatoria delle articolazioni sacroiliache [44].

Le forme cliniche di SA, se da una parte comprendono la spondilite anchilosante stabilizzata, dall'altra comprendono la early SA (uSpA), con il superamento dei limiti imposti dai criteri di New York modificati (1984) attraverso le nuove tecniche di imaging (RMN). HLA-B27 e RMN assumono un notevole valore diagnostico. Di qui la necessità di definire migliori criteri di classificazione, in cui includere la RMN, per intraprendere una terapia precoce, capace di modificare il decorso.

\section{Artrite psoriasica}

Si definisce come un reumatismo infiammatorio cronico, associato alla psoriasi, espressione del gruppo delle spondilo-enteso-artriti [56-59]. Malattia spesso progressiva e disabilitante, associata a una ridotta qualità di vita, presenta un'aumentata percentuale di morbilità e mortalità. Gli aspetti clinici sono polimorfi e comprendono:

- la poliartrite simmetrica, con un quadro sovrapponibile a quello dell'artrite reumatoide e assenza di noduli reumatoidi;

- l'artrite mutilante, con deformità articolari irregolari e bizzarre e prevalente interessamento delle piccole articolazioni di mani e piedi;

- l'artrite distale, con interessamento prevalente delle interfalangee distali;

- l'oligoartrite asimmetrica, con interessamento di meno di 5 articolazioni e decorso intermittente, con fasi di acuzie e di remissione;

- la spondiloartrite e la sacroileite, con quadri spesso indistinguibili dalla spondilite anchilosante [60-63].

La spondiloartropatia si presenta sotto forma di enteropatia e dattilite, e con caratteristiche specifiche, come la tendenza all'asimmetria, il coinvolgimento oligoarticolare, l'irite. Si parla di "early psoriatic arthritis" nel caso di oligoentesoartrite insorta da non più di 12 settimane [64-67].

Un intervento precoce richiede una diagnosi altrettanto precoce di artrite e/o spondilite.

L'onicopatia psoriasica interessa il 10-55\% dei pazienti con psoriasi e l' $80 \%$ dei pazienti con artropatia psoriasica. Nel $5 \%$ dei casi costituisce l'unica manifestazione; interessa matrice, letto, lamina o cute periungueale; si riscontrano pitting, oil spot, onicolisi, ipercheratosi subungueale, trachionichia [68].

\section{Conclusioni}

Le migliorate conoscenze patogenetiche, la maggiore disponibilità di più sensibili tecniche di imaging e il migliorato approccio terapeutico consentono di attuare terapie "targeted" efficaci, in grado di modificare la morbilità, la qualità di vita, la mortalità e, di conseguenza, il destino di tali pazienti.

\section{Conflitto di interesse}

Gli autori dichiarano di essere esenti da conflitto di interessi.

\section{Bibliografia}

[1] Brewerton DA, Hart FD, Nicholls A, Caffrey M, James DC, Sturrock RD. Ankylosing spondylitis and HL-A 27. Lancet 1973;1(7809):904-7.

[2] Moll JM, Haslock I, Macrae IF, Wright V. Associations between ankylosing spondylitis, psoriatic arthritis, Reiter's disease, the intestinal arthropathies, and Behcet's syndrome. Medicine (Baltimore) 1974;53(5):343-64.

[3] de Ceulaer K, van der Linden JM, Cats A. Sausage-like toes (dactylitis) and HLA B27. J Rheumatol Suppl 1977;3:66-9.

[4] Siegel DM, Baum J. HLA-B27 associated dactylitis in children. J Rheumatol 1988;15(6):976-7.

[5] Khan MA, van der Linden SM. A wider spectrum of spondyloarthropathies. Semin Arthritis Rheum 1990;20(2):107-13.

[6] Dougados $M$, van der Linden $S$, Juhlin R, et al. The European Spondylarthropathy Study Group preliminary criteria for the classification of spondylarthropathy. Arthritis Rheum 1991; 34(10):1218-27.

[7] Khan MA, editor. Spondyloarthropathies. Philadelphia, PA: WB Saunders; 1992.

[8] Khan MA. HLA-B27 and its subtypes in world populations. Curr Opin Rheumatol 1995;7(4):263-9.

[9] Brown MA, Pile KD, Kennedy LG, et al. HLA class I associations of ankylosing spondylitis in the white population in the United Kingdom. Ann Rheum Dis 1996;55(4):268-70.

[10] van der Linden S, van der Heijde D. Ankylosing spondylitis. Clinical features. Rheum Dis Clin North Am 1998;24(4):663-76.

[11] Khan MA. Spondyloarthropathies. In: Hunder G, editor. Atlas of Rheumatology. Philadelphia, PA: Current Science; 1998. p. 5.15.24 .

[12] McGonagle D, Khan MA, Marzo-Ortega H, O'Connor P, Gibbon W, Emery P. Enthesitis in spondyloarthropathy. Curr Opin Rheumatol 1999;11(4):244-50.

[13] François RJ, Gardner DL, Degrave EJ, Bywaters EG. Histopathologic evidence that sacroiliitis in ankylosing spondylitis is not merely enthesitis. Arthritis Rheum 2000;43(9):2011-24.

[14] Braun J, Khan MA, Sieper J. Enthesitis and ankylosis in spondyloarthropathy: what is the target of the immune response? Ann Rheum Dis 2000;59(12):985-94.

[15] Yu D. Spondylarthopathies. In: Ruddy S, Harris ED, Sledge CB, editors. Kelley's Textbook of Rheumatology. $6^{\text {th }}$ Ed., Philadelphia, PA: WB Saunders; 2000. p. 1039-53.

[16] Maksymowych WP. Ankylosing spondylitis. At the interface of bone and cartilage. J Rheumatol 2000;27(10):2295-301.

[17] Edwards JC, Bowness P, Archer JR. Jekyll and Hyde: the transformation of HLA-B27. Immunol Today 2000;21(6): 256-60.

[18] Cassidy JT, Petty RE, editors. Textbook of Pediatric Rheumatology. $4^{\text {th }}$ Ed., Philadelphia, PA: WB Saunders; 2001.

[19] François RJ, Braun J, Khan MA. Entheses and enthesitis: a histopathologic review and relevance to spondyloarthritides. Curr Opin Rheumatol 2001;13(4):255-64.

[20] Benjamin M, McGonagle D. The anatomical basis for disease localisation in seronegative spondyloarthropathy at entheses and related sites. J Anat 2001;199(Pt 5):503-26. 
[21] Laloux L, Voisin MC, Allain J, Martin N, Kerboull L, Chevalier X, et al. Immunohistological study of entheses in spondyloarthropathies: comparison in rheumatoid arthritis and osteoarthritis. Ann Rheum Dis 2001;60(4):316-21.

[22] Padula A, Giasi V, Olivieri I. Elderly onset isolated B27 associated dactylitis. Ann Rheum Dis 2002;61(8):759-60.

[23] Padula A, Scarano E, Giasi V, Olivieri I. Juvenile onset isolated HLA-B27-associated dactylitis. Semin Arthritis Rheum 2003; 32(5):341-2.

[24] Olivieri I, Scarano E, Padula A, Giasi V. Dactylitis involving most of the fingers. Clin Exp Rheumatol 2003;21(3):406.

[25] Olivieri I, Scarano E, Padula A, Giasi V, Priolo F. Dactylitis, a term for different digit diseases. Scand J Rheumatol 2006;35(5): $333-40$.

[26] Zeidler H, Mau W, Khan MA. Undifferentiated spondyloarthropathies. Rheum Dis Clin North Am 1992;18(1):187-202.

[27] Olivieri I, Padula A, Pierro A, Favaro L, Oranges GS, Ferri S. Late onset undifferentiated seronegative spondyloarthropathy. J Rheumatol 1995;22(5):899-903.

[28] Olivieri I, Salvarani C, Cantini F, Ciancio G, Padula A. Ankylosing spondylitis and undifferentiated spondyloarthropathies: a clinical review and description of a disease subset with older age at onset. Curr Opin Rheumatol 2001;13(4):280-4.

[29] Sampaio-Barros PD, Conde RA, Donadi EA, Shubin SV, Shoch BP, Donadi EA, et al. Undifferentiated spondyloarthropathies in Brazilians: importance of HLA-B27 and the B7-CREG alleles in characterization and disease progression. J Rheumatol 2003 Dec;30(12):2632-7.

[30] Olivieri I, van Tubergen A, Salvarani C, van der Linden S. Seronegative spondyloarthritides. Best Pract Res Clin Rheumatol 2002;16(5):723-39.

[31] McGonagle D, Gibbon W, Emery P. Classification of inflammatory arthritis by enthesitis. Lancet 1998;352(9134):1137-40.

[32] Olivieri I, Favaro L, Pierro A, Frisoni M, Ferri S, Pavlica P, et al. Dactylitis also involving the synovial sheaths in the palm of the hand. Ann Rheum Dis 1994;53(11):783-4.

[33] Olivieri I, Scarano E, Padula A, Giasi V. Dactylitis of the thumb extending to the radial bursa. J Rheumatol 2003;30(7):1626-7.

[34] McGonagle D, Pease C, Marzo-Ortega H, O'Connor P, Emery P. The case for classification of polymyalgia rheumatica and remitting seronegative symmetrical synovitis with pitting edema as primarily capsular/entheseal based pathologies. J Rheumatol 2000;27(4):837-40.

[35] Olivieri I, Salvarani C, Cantini F, Scarano E, Padula A, Niccoli L, et al. Fast spin echo-T2-weighted sequences with fat saturation in dactylitis of spondylarthritis. No evidence of entheseal involvement of the flexor digitorum tendons. Arthritis Rheum 2002;46(11):2964-7.

[36] Olivieri I, Scarano E, Padula A, Giasi V. Dactylitis of the thumb and the little finger extending to the carpal tunnel. J Rheumatol 2007;34(5):1155-6.

[37] McGonagle D, Marzo-Ortega H, Benjamin M, Emery P. Report on the Second international Enthesitis Workshop. Arthritis Rheum 2003;48(4):896-905.

[38] Healy PJ, Helliwell PS. Measuring dactylitis in clinical trials: which is the best instrument to use? J Rheumatol 2007;34(6):1302-6.

[39] Rudwaleit M, van der Heijde D, Khan MA, Braun J, Sieper J. How to diagnose axial spondyloarthritis early. Ann Rheum Dis 2004;63(5):535-43.

[40] Little H, Swinson DR, Cruickshank B. Upward subluxation of the axis in ankylosing spondylitis. A clinical pathologic report. Am J Med 1976;60(2):279-85.

[41] Calin A, Porta J, Fries JF, Schurman DJ. Clinical history as a screening test for ankylosing spondylitis. JAMA 1977;237(24): 2613-4.

[42] Amor B, Dougados M, Mijiyawa M. Criteria of the classification of spondylarthropathies. Rev Rhum Mal Osteoartic 1990;57(2): 85-9.
[43] Calin A, Taurog J. Spondylarthritides. New York, NY: Oxford University Press; 1998. p. 1-347.

[44] Rudwaleit M, Metter A, Listing J, Sieper J, Braun J. Inflammatory back pain in ankylosing spondylitis: a reassessment of the clinical history for application as classification and diagnostic criteria. Arthritis Rheum 2006;54(2):569-78.

[45] Zochling J, van der Heijde D, Burgos-Vargas R, Braun J, Burgos-Vargas D, Collantes R, et al., "ASsessment in $A S$ " international working group. European League Against Rheumatism. ASAS/EULAR recommendations for the management of ankylosing spondylitis. Ann Rheum Dis 2006;65(4): $442-52$.

[46] Sieper J, van der Heijde D, Landewé R, Brandt J, Hermann AG, Landewe K-G A, et al. New criteria for inflammatory back pain in patients with chronic back pain: a real patient exercise by experts from the Assessment of SpondyloArthritis international Society (ASAS). Ann Rheum Dis 2009;68(6): 784-8.

[47] Bennett AN, Marzo-Ortega H, Emery P, McGonagle D, Leeds Spondyloarthropathy Group. Diagnosing axial spondyloarthropathy. The new Assessment in SpondyloArthritis international Society criteria: MRI entering centre stage. Ann Rheum Dis 2009;68(6):765-7.

[48] Sieper J, Braun J. New treatment options in ankylosing spondylitis: a role for anti-TNFalpha therapy. Ann Rheum Dis 2001;60(Suppl 3):iii58-61.

[49] Braun J, Bollow M, Sieper J. Radiologic diagnosis and pathology of the spondyloarthropathies. Rheum Dis Clin North Am 1998;24(4):697-735.

[50] Olivieri I, Barozzi L, Favaro L, Pierro A, Frisoni M, Ferri F, et al. Dactylitis in patients with seronegative spondylarthropathy. Assessment by ultrasonography and magnetic resonance imaging. Arthritis Rheum 1996;39(9):1524-8.

[51] Olivieri I, Barozzi L, Pierro A, De Matteis M, Padula A, Pavlica P. Toe dactylitis in patients with spondyloarthropathy: assessment by magnetic resonance imaging. J Rheumatol 1997;24(5): 926-30.

[52] Wakefield RJ, Emery P, Veale D. Ultrasonography and psoriatic arthritis. J Rheumatol 2000;27(6):1564-5.

[53] Padula A, Salvarani C, Barozzi L, De Matteis M, Olivieri I, Favaro $\mathrm{L}$, et al. Dactylitis also involving the synovial sheaths in the palm of the hand: two more cases studied by magnetic resonance imaging. Ann Rheum Dis 1998;57(1):61-2.

[54] McGonagle D, Gibbon W, O'Connor P, Green M, Pease C, Emery P. Characteristic magnetic resonance imaging entheseal changes of knee synovitis in spondylarthropathy. Arthritis Rheum 1998;41(4):694-700.

[55] Healy PJ, Groves C, Chandramohan R, Helliwell PS, Vena GA, Galluccio A, et al. Magnetic resonance imaging in dactylitis. What is the site of inflammation? [abstract] Ann Rheum Dis 2006;65(Suppl 2):68.

[56] Wright V. Psoriatic arthritis. In: Scott JT, editor. Copeman's Textbook of the Rheumatic Diseases. $5^{\text {th }}$ Ed., Edinburgh: Churchill Livingstone; 1978 . p. 537-48.

[57] Bennett RM. Psoriatic arthritis. In: McCarty DJ, editor. Arthritis and Allied Conditions. $9^{\text {th }}$ Ed, Philadelphia, PA: Lea and Febiger; 1979. p. 642-55.

[58] Bennett RM. Psoriatic arthritis. In: McCarty DJ, editor. Arthritis and Allied Conditions. $10^{\text {th }}$ Ed., Philadelphia, PA: Lea and Febiger; 1985. p. 850-66.

[59] Gladman DD. Psoriatic arthritis. In: Khan MA, editor. Ankylosing Spondylitis and Related Spondyloarthropathies. Philadelphia, PA: Hanley and Belfus; 1990. p. 637-56.

[60] Taylor W, Gladman D, Helliwell P, Marchesoni A, Mease P, Mielants H, CASPAR Study Group. Classification criteria for psoriatic arthritis: development of new criteria from a large international study. Arthritis Rheum 2006;54(8):266573. 
[61] Salvarani C, Cantini F, Olivieri I, Macchioni P, Niccoli L, Padula A, et al. Isolated peripheral enthesitis and/or dactylitis: a subset of psoriatic arthritis. J Rheumatol 1997;24(6):1106-10.

[62] Padula A, Belsito F, Barozzi L, Cantini F, Salvarani C, Pavlica P, et al. Isolated tenosynovitis associated with psoriasis triggered by physical injury. Clin Exp Rheumatol 1999;17(1):103-4.

[63] Scarpa R, Soscia E, Peluso R, Oriente A, Atteno M, Manguso F, et al. Nail and distal interphalangeal joint in psoriatic arthritis. J Rheumatol 2006;33(7):1315-9.

[64] Kane D, Greaney T, Bresnihan B, Gibney R, FitzGerald O. Ultrasonography in the diagnosis and management of psoriatic dactylitis. J Rheumatol 1999;26(8):1746-51.
[65] Brockbank JE, Stein M, Schentag CT, Gladman DD. Dactylitis in psoriatic arthritis: a marker for disease severity? Ann Rheum Dis 2005;64(2):188-90.

[66] Kavanaugh AF, Ritchlin CT, GRAPPA Treatment Guideline Committee. Systematic review of treatments for psoriatic arthritis: an evidence based approach and basis for treatment guidelines. J Rheumatol 2006;33(7):1417-21.

[67] Helliwell PS. Therapies for dactylitis in psoriatic arthritis. A systematic review. J Rheumatol 2006;33(7):1439-41.

[68] Helliwell PS, Firth J, Ibrahim GH, Melsom RD, Shah I, Turner DE. Development of an assessment tool for dactylitis in patients with psoriatic arthritis. J Rheumatol 2005;32(9):1745-50. 